\title{
A influência do desempenho motor no "status" social percebido por crianças
}

CDD. 20.ed. 152.3

613.7

796.05

http://dx.doi.org/10.1590/1807-55092016000401069

\begin{tabular}{|c|c|}
\hline $\begin{array}{l}\text { Pâmella MEDEIROS* } \\
\text { Marcela Almeida ZEQUINÃO* } \\
\text { Fernando Luiz CARDOSO* }\end{array}$ & $\begin{array}{l}\text { "Centro de Ciências } \\
\text { da Saúde e do Es- } \\
\text { porte, Universidade } \\
\text { do Estado de Santa } \\
\text { Catarina, Florianópolis, } \\
\text { SC, Brasil. }\end{array}$ \\
\hline
\end{tabular}

\section{Resumo}

Este estudo teve como objetivos descrever o desempenho motor de crianças e adolescentes em relação ao "status" social dos mesmos segundo a percepção dos colegas nos diferentes sexos, além de verificar se há o efeito sexo nessa possivel associação. Participaram desta pesquisa 300 crianças e adolescentes do $3^{\circ}$ ao $5^{\circ}$ ano e da $4^{a}$ a $6^{a}$ série do Ensino Fundamental de duas escolas públicas, com idades entre oito e 14 anos. Os instrumentos utilizados foram a Bateria motora TGMD-2 e a Escala Subjetiva de Status Social. As meninas apresentaram melhor desempenho motor que os meninos, no entanto, apenas os meninos com melhor desempenho motor também apresentaram maior "status" social percebido. Tal situação nos indica que os meninos estão mais suscetiveis a avaliações sociais positivas pautadas em competências físicas que as meninas, podendo fazer uso do seu melhor nível de desempenho motor para elevar a sua popularidade na escola através de práticas esportivas.

Palavras-chave: Destreza motora; Hierarquia social; Educação física; Fatores sexuais.

\section{Introdução}

O desenvolvimento motor é o processo de maturação do sistema motor ao longo do tempo, compreendendo modificaçóes contínuas do comportamento motor, realizado pela influência mútua entre as necessidades da tarefa, a biologia do indivíduo e a interação com o meio ambiente, que combinados resultam em uma melhora do desempenho que representa a aquisição de funçóes motoras cada vez mais complexas ${ }^{1}$.

Os parâmetros normais de desenvolvimento motor servem como padróes típicos do desenvolvimento infantil, representando a idade média em que a criança é capaz de realizar habilidades motoras básicas, que são requisitadas no seu cotidiano, sendo imprescindíveis para o diagnóstico do desenvolvimento infantil ${ }^{2-3}$. Os aspectos motores apresentados na infância sofrem modificaçôes com a idade e são inerentes às diferenças sociais, intelectuais e emocionais ${ }^{4}$.

Dessa forma, crianças e adolescentes que apresentam movimentos precisos, adequados e que manifestam respostas rápidas a uma determinada situação, possuem maiores probabilidades de sucesso no desempenho de suas tarefas motoras ${ }^{5}$, o que tende a causar efeitos positivos em outros domínios da vida diária, como nos relacionamentos intra einterpessoal ${ }^{6}$, refletindo no sucesso das relaçóes do contexto escolar.

No universo infanto-juvenil as comparaçōes sociais, a avaliação do desempenho motor dos colegas e a avaliaçáo que recebem de terceiros, influenciam de forma efetiva no desenvolvimento do senso de auto eficácia, responsável pela promoção da satisfação e sustentaçáo de relacionamentos sociais positivos, influenciando seu "status" social perante o grupo ${ }^{7-8}$. Este, por sua vez pode ser entendido como a posiçấo social de uma pessoa frente às percepções dos seus pares nos diferentes níveis sociais em que convive?, podendo ter impacto negativo ou positivo na vida dos indivíduos ${ }^{10}$, principalmente na infância e adolescência, nos quais meninas e meninos são mais dependentes da aceitação e aprovação dos pares ${ }^{11-14}$.

$\mathrm{Na}$ escola, o grau de "status" que o aluno possui perante o grupo, seja na disputa pelo poder ou na marcação territorial ${ }^{15}$, pode estar associada a algumas características como idade, tempo que 
está na instituição ${ }^{16-18}$, ser bom em esportes e até mesmo o nível de desempenho motor que ele apresenta ${ }^{19}$. Estudos vêm apontando que crianças e adolescentes que apresentam um melhor nível de desempenho motor e um maior nível de atividade física, consequentemente também apresentam um maior "status" social percebido pelo grupo ${ }^{20-23}$. No entanto, os alunos com nível mais baixo de desempenho motor, pouca habilidade para os jogos com bola, são constantemente excluídos do grupo, das brincadeiras e jogos nos recreios e nas aulas de Educação Física, enquanto os mais aptos tendem a dominar ${ }^{20,22,24-26}$. Com isso, muitas crianças se retiram dessas situaçóes para evitarem demonstrar qualquer proficiência motora abaixo da média. Esse pode se tornar um problema ainda maior e de efeitos relativamente duradouros, não apenas pelo

\section{Método}

Esta é uma pesquisa descritiva, transversal e de campo que procurou por possíveis correlações entre o nível de desempenho motor e o "status" social segundo a percepção dos próprios colegas.

\section{Participantes}

Participaram do estudo 300 crianças e adolescentes do $3^{\circ}$ ao $5^{\circ}$ ano e da $4^{\mathrm{a}}$ a $6^{\mathrm{a}}$ série do Ensino Fundamental, de ambos os sexos, com idades entre oito e 14 anos, matriculadas em duas escolas públicas municipais no período vigente do estudo. Esta pesquisa faz parte de um projeto aprovado junto ao Comitê de Ética em Pesquisas com Seres Humanos sob processo 5439/2011, n. expediente 75/2011.

\section{Instrumentos}

Para a avaliação do nível de desenvolvimento motor utilizou-se a bateria Test of Gross Motor Development- Second Edition-TGMD-2 ${ }^{31}$, que consiste em uma avaliação normativa das habilidades motoras globais consideradas comuns, referenciada por norma e critério ${ }^{44}$. É composto por 12 habilidades motoras fundamentais, divididas em dois sub-testes específcos: habilidades de locomoção (correr, galopar, saltitar, saltar obstáculo, saltar horizontalmente e deslizar); e habilidades de controle de objetos (rebater, quicar, receber, chutar, arremessar por cima do ombro e rolar prejuízo no desenvolvimento motor, mas também pelo prejuízo nas relaçóes pessoais ${ }^{27-30}$.

No entanto, pouco se sabe como a relação das variáveis desempenho motor e "status" social pode atuar em meninos e meninas separadamente. $\mathrm{O}$ entendimento dessa relação pode tornar-se mais complexo quando confrontamo-la com a variável identidade sexual, ou seja, até que ponto ser menino ou menina com melhor ou pior desempenho motor pode interferir nas estratégias escolhidas para se ganhar mais "status" na escola?

Diante disso, o presente estudo tem como objetivos descrever o desempenho motor de crianças e adolescentes em relação ao "status" social dos mesmos segundo a percepção dos colegas nos diferentes sexos, e por fim verificar se há relação destas variáveis em meninos e meninas separadamente.

a bola). Em cada habilidade são observados de três a cinco critérios motores específicos que permitem avaliar como as crianças coordenam o tronco e membros durante o desempenho de uma habilidade motora, ou seja, analisa o padrão da habilidade motora, isto é, o processo e náo o produto final da tarefa.

Cada habilidade foi executada duas vezes, sendo as duas avaliadas e pontuadas. Cada sub-teste possui 24 critérios de desempenho cada um e em cada critério é atribuída uma nota 0 (não atendeu ao critério) ou 1 (atendeu ao critério), podendo totalizar um máximo de dois pontos por critério em cada habilidade. Desta forma, cada criança avaliada pode ter no máximo 48 pontos no escore de cada sub-teste. A partir desta pontuação é necessário consultar o manual do teste proposto por ULRICH ${ }^{31}$ para determinar o Quociente Motor Bruto, pois cada criança recebe um escore conforme a idade e o sexo para cada sub-teste. Assim, determina-se o nível de desempenho da mesma, classificando-a em sete categorias: "muito pobre, pobre, abaixo da média, na média, acima da média, superior e muito superior" 31 .

Para verificar o "status" social segundo a percepção dos colegas, utilizou-se o método sociométrico ${ }^{32}$, que consiste em analisar a aceitação ou rejeição social por meio de escolhas sociométricas positivas ou negativas ${ }^{33}$. Aplicou-se então, a Escala Sociométrica de Status Social Subjetiva de Levandoski ${ }^{16}$, composto por seis questôes de avaliação de um aluno em relação aos seus colegas, através de indicações 
de acordo com seis habilidades específicas percebidas através do convívio diário: três questôes que abrangem o "status" positivo Q1 (Primeiros a serem escolhidos na Educação Física), Q3 (Mais fortes) e Q5 (Primeiros a serem escolhidos para trabalhos em sala de aula) e três questóes de "status" negativo Q2 (Últimos a serem escolhidos na Educaçâo Física), Q4 (Mais fracos) e Q6 (Últimos a serem escolhidos para trabalhos em sala de aula). O aluno consultado pode indicar até 10 colegas para cada pergunta independente do $\operatorname{sexo}^{16}$. Ao final foi realizada uma contagem de frequência com os alunos indicados, obtendo assim, escores para cada um, tanto para "status" positivo, quanto negativo. Estudos como Core et al..$^{34}$, enfatizam a importância de se usarem o método sociométrico, obtendo com a combinação de questóes positivas e negativas, resultados mais precisos envolvendo toda a esfera do "status" social.

\section{Procedimentos}

O teste foi filmado individualmente e as filmagens analisadas por três avaliadores, sendo comparadas as notas dadas em cada critério 0 (não atendeu ao critério) ou 1 (atendeu ao critério), de modo que o resultado considerado fosse indicado por pelo menos dois dos avaliadores em cada critério e em cada tentativa. A aplicação do teste motor TGMD-2 foi realizada em um espaço apto com piso de concreto, cedido pelas escolas, de forma individual, sob a orientaçấo de dois

\section{Resultados}

\section{Desempenho motor e sua relação com o sexo dos participantes}

Quando analisados separadamente os escores das Habilidades Locomotoras e de Controle de Objetos em relação aos sexos, verificou-se que meninas apresentaram melhor nível de desempenho motor em relação aos meninos nas habilidades de controle de objeto $(\wp=6,96 ; \mathrm{DP}=2,24 ; \mathrm{p} \leq 0,001)$. Da mesma forma, quando analisado o Quociente Motor Bruto, as meninas também foram as que pesquisadores treinados e capacitados para a aplicação: um foi responsável por instruir os participantes, e outro por filmar as habilidades. Já o questionário para avaliar o "status" social na percepção dos colegas, foi aplicado individualmente em uma sala de aula por um pesquisador treinado e capacitado. Ambos os testes foram aplicados no ambiente escolar.

\section{Análise estatística}

Os dados da pesquisa foram tabulados e analisados no programa computadorizado Statistical Package for the Social Science (SPSS for Windows) versão 20.0, mantendo a identidade das crianças em sigilo. Para análise dos dados utilizou-se o teste de normalidade de KolmogorovSmirnov para verificar se os dados atendiam os pressupostos paramétricos. Como os dados não apresentaram distribuição normal, realizou-se a estatística descritiva e inferencial por meio dos testes Qui-quadrado, para verificar a associação entre o desempenho motor categorizado e a variável sexo; Mann-Whitney, para comparação entre os sexos em relação aos escores de desempenho motor e o "status" percebido, e correlação de Spermann para verificar a relação entre os escores de desempenho motor e "status" percebido pelos colegas. Utilizou-se, também, o programa $\mathrm{G}$ power 3.1, para calcular o tamanho do efeito da correlação entre o desempenho motor e o "status" social dos participantes segundo a percepçáo dos colegas.

apresentaram melhores resultados $(\wp=81,45$; DP $=10,70 ; \mathrm{p} \leq 0,007)$ - TABELA 1 .

Apesar das meninas apresentarem escores superiores no geral em relação aos meninos, quando os participantes foram classificados em quatro categorias (muito pobre, pobre, abaixo da média e na média), não foram encontradas associaçôes entre estas e o sexo masculino e feminino. Além disso, pelas frequências foi possível observar que nenhum dos alunos atingiu os níveis acima da média, superior e muito superior, conforme a TABELA 2. 
TABELA 1 - Escores dos sub-testes e do quociente motor bruto, controladas pelo sexo dos participantes.

${ }^{*} \chi$ : média;

DP: Desvio padrão;

p: nível de significância

$p \leq 0,05$;

U: teste de Mann Whi-

tney.

\begin{tabular}{lccccc}
\hline \multirow{2}{*}{ Variáveis } & $\begin{array}{c}\text { Meninos } \\
(\mathbf{n = 1 5 2})\end{array}$ & $\begin{array}{c}\text { Meninas } \\
(\mathbf{n}=\mathbf{1 4 8})\end{array}$ & $\mathbf{U}$ & $\mathbf{p} \leq$ \\
\cline { 2 - 3 } & $\begin{array}{lccc}\chi(\mathrm{dp}) \\
\text { Escore habilidades locomotoras }\end{array}$ & $6,79(2,17)$ & $6,80(2,12)$ & & \\
Escore habilidades de controle de objetos & $6,10(2,31)$ & $6,96(2,24)$ & 9001,50 & 0,001 \\
Quociente motor bruto & $78,78(9,89)$ & $81,45(10,70)$ & 9243,00 & 0,007 \\
\hline
\end{tabular}

TABELA 2 -Associação o desempenho motor categorizado e o sexo dos participantes.

${ }^{*} \mathrm{~N}$ : número de participantes;

\%: frequência;

$\chi^{2}$ : Teste do qui qua-

drado;

p: nível de significância $p \leq 0,05$.

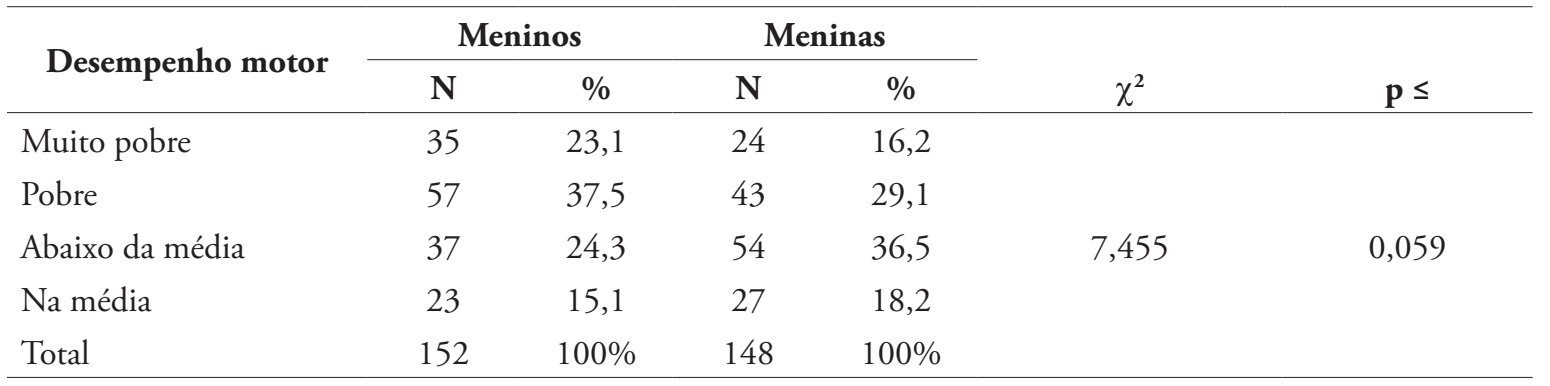

"Status" na percepção dos colegas: diferença entre os sexos

Em relação ao "status" social segundo a percepção dos colegas, identificou-se que os meninos foram mais vezes escolhidos preferencialmente nas aulas de Educação Física, foram também mais vezes citados como os mais fortes e mais fracos, além de serem os últimos a serem escolhidos para trabalhos em dupla realizados em sala de aula, quando comparados às meninas. Em contrapartida, as meninas foram mais vezes citadas como as preferidas para fazerem os trabalhos escolares em sala de aula com o grupo.

Em relação ao escore de "status" positivo, os meninos possuem um maior "status" pela percepçáo dos seus colegas. Contudo, quando somadas às questóes que fazem referência a um pior "status", também foram os meninos aqueles mais vezes indicados como tendo menor "status" segundo a percepção dos colegas, quando comparados as meninas (TABELA 3).

TABELA 3 - Diferenças dos escores do "status" segundo a percepção dos colegas com os sexos dos participantes.

${ }^{*} \chi$ : média extraídado número de indicações pelos pares, quanto maior os números, maior o número de indicações;

DP: desvio padrão;

U: teste de Mann Wi-

thney;

p: nível de significância $p \leq 0,05$.

\begin{tabular}{lcccc}
\hline \multirow{2}{*}{ Questóes } & $\begin{array}{c}\text { Meninos } \\
(\mathbf{n = 2 0 7})\end{array}$ & $\begin{array}{c}\text { Meninas } \\
(\mathbf{n = 2 0 2})\end{array}$ & $\mathbf{U}$ & $\mathbf{p} \leq$ \\
\cline { 2 - 3 } & $\chi(\mathbf{d p})$ & $\chi(\mathbf{d p})$ & & \\
\hline Q1 Primeiros escolhidos na Educação Física & $\mathbf{5 , 3 7 ( 4 , \mathbf { 1 2 } )}$ & $4,24(2,79)$ & 16260,00 & $\mathbf{0 , 0 1 3}$ \\
Q2 Últimos escolhidos na Educação Física & $3,05(2,39)$ & $2,55(2,02)$ & 16904,00 & 0,055 \\
Q3 Mais fortes & $\mathbf{3 , 5 5 ( 4 , 3 2 )}$ & $1,17(1,84)$ & 11100,00 & $\mathbf{0 , 0 0 1}$ \\
Q4 Mais fracos & $\mathbf{2 , 7 3 ( 2 , 6 3 )}$ & $2,02(2,14)$ & 15889,50 & $\mathbf{0 , 0 0 4}$ \\
Q5 Primeiros escolhidos para sala/aula & $2,14(4,32)$ & $\mathbf{3 , 1 5 ( 2 , 3 3 )}$ & 14044,50 & $\mathbf{0 , 0 0 1}$ \\
Q6 Últimos escolhidos para sala/aula & $\mathbf{2 , 9 7 ( 2 , 0 4 )}$ & $1,63(1,59)$ & 12417,50 & $\mathbf{0 , 0 0 1}$ \\
Escore "status" positivo & $\mathbf{1 1 , 0 7 ( 1 0 , 0 0 )}$ & $\mathbf{8 , 5 5 ( 5 , 6 0 )}$ & $\mathbf{1 6 1 8 5 , 5 0}$ & $\mathbf{0 , 0 1 1}$ \\
Escore "status" negativo & $\mathbf{8 , 7 5 ( 5 , 5 0 )}$ & $\mathbf{6 , 1 9 ( 4 , 5 2 )}$ & $\mathbf{1 3 2 2 3 , 0 0}$ & $\mathbf{0 , 0 0 1}$ \\
\hline
\end{tabular}




\section{Relação entre o desempenho motor e "status" social entre os sexos, segundo a percepção dos colegas}

Ao analisar as relaçóes entre o desempenho motor e o "status" dos participantes segundo a percepção dos colegas foi encontrada uma correlação positiva $\left.\rho=0,274^{* *} ; p \leq 0,001\right)$ entre "status" positivo na percepção dos colegas e o desempenho motor, por meio do quociente motor bruto, para os meninos. Isto é, quanto melhor o nível de desempenho motor do aluno, mais popular na visão dos colegas. Já para as meninas, nenhuma correlação foi encontrada ( $\rho=$ -0,001; $p=0,995)$. Tamanho do efeito: 0,29.

A FIGURA 1 ilustra a associação entre as duas variáveis utilizando os escores brutos: desempenho motor e o "status" dos participantes.

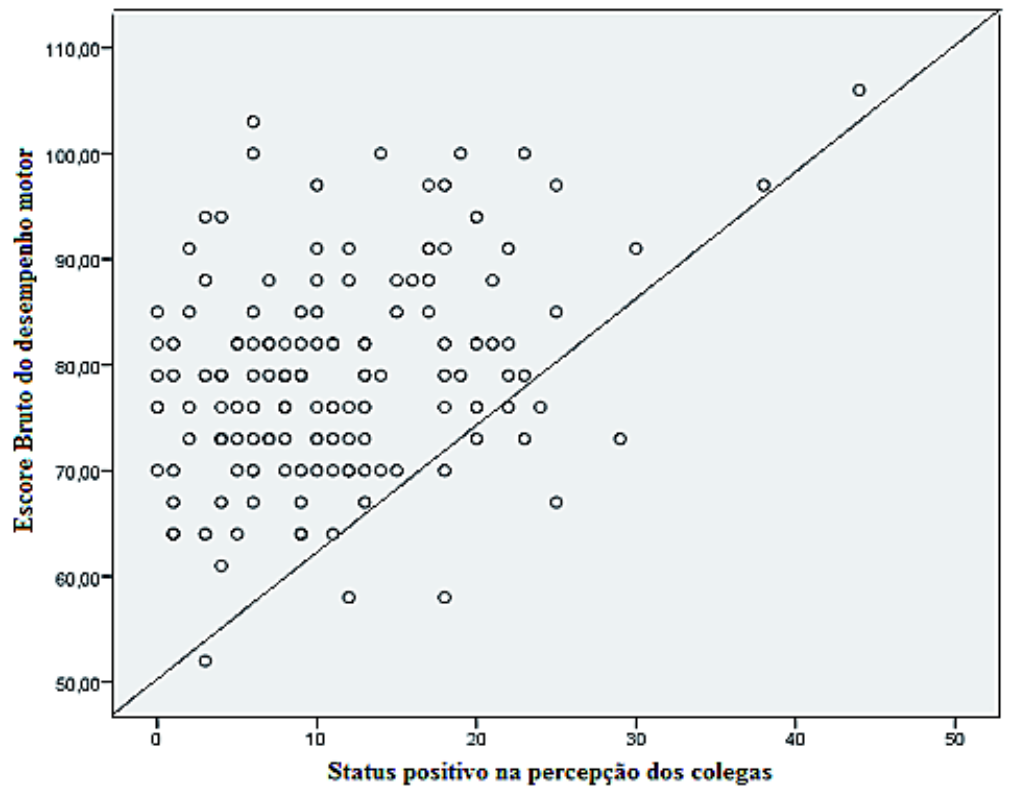

FIGURA 1 - Gráfico de correlação entre desempenho motor e "status" na percepção dos colegas.

\section{Discussão}

\section{Desempenho motor e sua relação com o sexo dos participantes}

Dentro de um contexto desenvolvimentista, as habilidades motoras fundamentais são aquelas que envolvem os grandes grupos musculares do tronco, braços e pernas. Essas habilidades envolvem tarefas de locomoção e manipulação, e quando desenvolvidas possibilitam o aperfeiçoamento para tarefas especializadas como: transportar o corpo pelo espaço e explorar e interagir com os objetos no ambiente ${ }^{35}$. Tais habilidades podem ser utilizadas em situaçóes específicas de esporte, dança e atividades lúdicas ${ }^{36} \mathrm{e}$ assim, construir um diversificado repertório motor, possibilitando que os desafios do cotidiano sejam enfrentados de maneira adequada e efetiva ${ }^{37}$.

Em geral, testes que avaliam essas habilidades, têm mostrado que meninos em idade escolar possuem significativamente melhor desempenho motor em relação às meninas ${ }^{38-42}$. Além disso, BlatCHORD et al ${ }^{43}$ relataram que meninos são mais propensos a se envolverem em jogos com bola o que pode levá-los a níveis mais altos de atividades físicas e melhores habilidades manipulativas. Esses dados, vão de encontro aos resultados deste estudo, no qual meninas apresentaram melhor desempenho nas habilidades de controle de objetos e no quociente motor bruto quando comparadas aos meninos. Esse resultado foi semelhante aos achados de Авіко et al. ${ }^{44}$, também com estudantes da regiáo Sul do Brasil, em que, do mesmo modo, meninas apresentaram resultados superiores aos meninos no resultado geral, embora a idade dos participantes das pesquisas tenha sido inferior aos do presente estudo, no entanto maiores especificidades em relaçáo ao contexto social não foi encontrado nos estudos. 
Ainda que meninas tenham apresentado melhores resultados quando comparadas aos meninos, verificou-se que, em ambos os sexos, os níveis de desempenho motor foram muito baixos em geral, sendo que apenas $15,1 \%$ dos meninos e $18,2 \%$ das meninas atingiram o nível médio, ficando todos os outros participantes em níveis inferiores. Esses dados indicam um grande atraso no desenvolvimento motor das crianças e adolescentes deste estudo, tendo em vista que a literatura vem apontando que no decorrer do crescimento e do desenvolvimento infantil a habilidade em usar os segmentos corporais deveria aumentar em força, velocidade e coordenaçãa ${ }^{35,45-47}$.

\section{"Status" na percepção dos colegas: diferença entre os sexos}

Quando analisados neste estudo o "status" percebido pelos colegas em quase todas as questôes os meninos foram mais lembrados que as meninas, tanto para perguntas sobre "status" positivo, quanto negativo, indo ao encontro de estudos como o de Core et al. ${ }^{34}$ e Morais et al. ${ }^{33}$. Em contrapartida, as meninas foram as mais citadas como sendo as primeiras escolhidas para fazerem trabalhos em sala de aula. Isso se justifica tendo em vista que diversos estudos têm mostrado os meninos com um pior desempenho escolar e maiores dificuldades para aprender ${ }^{48-52}$.

Apesar de os meninos apresentarem um desempenho superior às meninas em matemática, na língua portuguesa acontece ao contrário, sendo este padrão recorrente em grande parte das sociedades estudadas ${ }^{53}$. Embora existam essas variações para maiores facilidades e dificuldades em cada disciplina, estudos apontam uma incidência de três meninos para uma menina em relação às dificuldades para aprender, podendo variar ainda em até 10 para um ${ }^{48-50,54-55}$.

A relação entre "status" e habilidades cognitivas espelha a influência dos tradicionais estereótipos de gênero já muito bem documentado na literatura ${ }^{56}$. Nas relaçóes que a criança cria com o grupo, ela estabelece estratégias para exercer o poder, assim como mecanismos para obter a aceitação e sua posição no grupo $^{33}$. OsTBERG ${ }^{57}$ verificou que durante a infância, quanto menor sua posição de "status" percebidos pelos colegas em relação aos seus pares, o indivíduo apresentará uma menor auto avaliaçấo de saúde na vida adulta, além de influenciar sintomas de estresse emocional e açóes de vitimização de "bullying" escolar durante a infância e adolescência. Crianças e adolescentes com um menor "status" social correm sérios riscos de desenvolverem problemas de ajustamento social tanto no presente como no futuro ${ }^{33,58}$.

\section{Desempenho motor e sua relação com o "status" social}

Neste estudo, foi encontrada uma associação entre ter melhor desempenho motor e maior "status" social segundo a percepçáo dos colegas, entre os meninos. Esses dados estáo de acordo com a literatura que considera o nível de desempenho motor influenciador no modo como às crianças se percebem e percebem seus pares e, quando experimentadas sensaçóes de divertimento, satisfação e sucesso nas atividades físicas, o nível de autoestima e motivaçáo tendem a se elevar ${ }^{35,59}$. Além disso, para Oldehinkelet al. ${ }^{19}$ um melhor desempenho motor está associado a um maior "status" social em crianças adolescentes. Isso se aplica principalmente para os meninos, que estão mais suscetíveis a avaliaçóes que as meninas, pois estudos indicam os meninos como mais competitivos por dominância social e "status" 60 estando mais expostos à avaliação dos colegas. Além disso, muitas meninas reforçam essa competitividade entre os meninos, fazendo com que eles se tornem, inclusive, mais agressivos, pois para elas os meninos agressores são os alunos mais populares da sala e que se destacam em atividades de força e habilidades esportivas, bem como as crianças que não se destacam são vistas em menor posição social ${ }^{61}$.

O sucesso nas atividades físicas, o envolvimento em esportes em idade escolar e o reconhecimento de seus pares pode aumentar a popularidade, bem como restringir as possibilidades de ser intimidado na escola $^{62}$. Segundo WeInECK ${ }^{63}$ crianças e adolescentes que possuem um amplo conhecimento motor são cobiçados parceiros de jogos, sendo aqueles que sabem correr rápido, pegarem bem uma bola e serem ágeis, os mais bem-vistos por seu grupo social. No entanto, o contrário também é verdadeiro, sendo que crianças e adolescentes com menos habilidades motoras e um fraco desempenho em jogos tendem a ter menos amigos, serem rejeitadas pelos pares, são menos convidadas para brincar e participar de jogos ${ }^{26,28,64-65}$. Assim, pode-se considerar que o desempenho motor na infância e adolescência é fundamental para a percepção e os sentimentos que os indivíduos têm de si, principalmente em função do "status" social que este pode proporcionar aos mesmos ${ }^{37}$.

Desta forma conclui-se que embora as meninas tenham apresentado um melhor desempenho motor geral em relação os meninos, encontrou-se uma 
associação entre maior "status" percebido pelos colegas e um melhor desempenho motor apenas para os meninos. Isso nos dá indícios de que os meninos são mais suscetíveis a serem avaliados socialmente a partir da sua motricidade. Essa diferença entre meninos e meninas poderia ser explicada por questôes relacionadas à identidade de gênero dos participantes, no entanto este estudo limitou-se apenas a avaliar o sexo dos mesmos. Assim, sugere-se que mais estudos sejam realizados para testar tal hipótese controlando o gênero dos participantes, isto é uma orientação para a masculinidade ou feminilidade.

Esta associação entre melhor desempenho motor e maior "status" social segundo a percepção dos colegas para os meninos pode trazer grandes contribuiçóes para uma Educação Física mais inclusiva, à medida que os professores possam criar estratégias metodológicas que resgatem os meninos com menor "status" e controlem os meninos com maior "status", para que não façam uso de seu poder social de maneira negativa com os pares. A confirmação dessa associação entre essas duas variáveis fortemente influenciadas pela identidade sexual dos participantes pode fundamentar uma prática de Educação Física desenvolvimentista, inclusiva e responsável, pois as habilidades motoras trabalhadas na escola podem influenciar comportamentos positivos e negativos no ambiente educacional quando respeitadas as diferenças de sexo.

\section{Limitações}

Apesar da relevante contribuição deste estudo em produzir novos dados quantitativos para melhor explicar e náo só interpretar o desenvolvimento motor e humano, este estudo foi realizado em um ambiente especifico que se caracteriza por uma alta vulnerabilidade social, nos impedindo de fazer qualquer generalização das nossas conclusóes para a população escolar catarinense como um todo. Mais estudos comparativos sobre a influência da marginalização social em escolares em termos de desenvolvimento motor, desempenho escolar, "status" social e identidade de gênero / sexo são necessários e urgentes.

\section{Abstract}

\section{Motor performance influence in social status perceived}

This study aimed to describe the children and adolescents motor performance in relation to their social status perceived by their colleagues in both sexes, and to check for sex effects in this possible association. The study gathered 300 children and adolescents from the $3 \mathrm{rd}$ to 5 th year and $4^{0}$ to $6^{0}$ grade of elementary school from two public schools, aged between 8 and 14 years. The instruments used were the Motor Battery TGMD-2 and Subjective Scale Social Status. Girls had better motor performance than boys, however, only boys with better motor performance also had higher perceived social status. This situation tells us that boys are more susceptible to social evaluations guided in physical skills than girls so that they can make use of their best motor performance level to increase their popularity at school through sports practices.

Key Words: Motor skills; Hierarchy; Physical education; Sex factors.

\section{Referências}

1. Gallahue D, Ozmun J. Compreendendo o desenvolvimento motor: bebês, crianças, adolescentes e adultos. São Paulo: Phorte; 2005.

2. Tani G. Educação física na pré-escola e nas quatro primeiras séries do ensino de primeiro grau: uma abordagem de desenvolvimento. Kinesis. 1987;3:19-41.

3. Santos S, Dantas L, Oliveira J. Desenvolvimento motor de crianças, de idosos e de pessoas com transtornos da coordenação. Rev Paul Educ Fís. 2004;18:33-44.

4. Payne V, Isaacs L. Desenvolvimento motor humano: uma abordagem vitalícia. Rio de Janeiro: Guanabara: Koogan; 2007.

5. Hemgren E, Persson K. Deficits in motor co-ordination and attention at 3 years of age predict motor deviations in 6.5-year-old children who needed neonatal intensive care. Child Care Health Dev. 2009;35:120-9.

6. Emck, C, Bosscher R, Beek P, Doreleijrs T. Gross motor performance and self-perceived motor competenceinchildren with emotional, behavioural, and pervasive developmental disorders: a review. Dev Med Child Neurol. 2009;51:501-17. 
7. Bandura A. Human agency in social cognitive theory. Am Psychol. 1989;44:1175-84.

8. Medeiros P, Loureiro SR, Linhares MBM, Marturano EM. A auto-eficácia e os aspectos comportamentais de crianças com dificuldade de aprendizagem. Psicol Reflex Crít. 2000;13:327-36.

9. Singh-Manoux A, Marmot MG, Adler NE. Does subjective social status predict health and change in health status better than objective status? Psychosom Med. 2005;67:855-61.

10. Macleod J, Davey Smith G, Metcalfe C, Hart C. Is subjective social status a more important determinant of health than objective social status? Evidence from a prospective observational study of Scottish men. Soc Sci Med. 2005;61:1916-29.

11. Presnell K, Bearman SK. Risk factors for body dissatisfaction in adolescent boys and girls: a prospective study. Int J Eat Disord. 2004;36:389-401.

12. Gerner B, Wilson PH. The relationship between friendship factors and adolescent girls' body image concern, body dissatisfaction, and restrained eating. Int J Eat Disord. 2005;37:313-20.

13. Bearman SK, Martinez E, Stice E, Presnell K. The skinny on body dissatisfaction: a longitudinal study of adolescent girls and boys. J Youth Adolesc. 2006;35:217-29.

14. Ata R, Ludden A, Lally M. The effects of gender and family, friend, and media influences on eating behaviors and body image during adolescence. J Youth Adolesc. 2007:36:1024-37.

15. Morris D. O macaco nu: um estudo do animal humano. Rio de Janeiro: Record; 1967.

16. Levandoski G. Análise de fatores associados ao comportamento bullying no ambiente escolar: características cineantropométricas e psicossociais [dissertação]. Florianópolis (SC): Universidade do Estado de Santa Catarina; 2009.

17. Crandall C. Prejudice against fat people: ideology and self-interest. J Pers Soc Psychol. 1994;66:882-94.

18. Crosnoe R, Frank K, Mueller A. Gender, body size and social relations in American high schools. Soc Forces. 2008;86.

19. Oldehinkel A, Rosmalen JGM, Veenstra R, Dijkstra JK, Ormel J. Being admired or being liked: classroom social status and depressive problems in early adolescent girls and boys. J Abnorm Child Psychol. 2007;35:417-27.

20. Higgins C. How to improve the school ground enviroment as an antibullying strategy. In: Sharp S, Smith P, editors. Tackling bullying in your school. London: Routledge; 1994. p.133-73.

21. Wolke D, Woods S, Stanford K, Schulz H. Bullying and victimization of primary school children in England and Germany: prevalence and school factors. Br J Psychol. 2001;92: 673-96.

22. Botelho R, Souza J. Bullying e educação física na escola: características, casos, consequências e estratégias de prevenção. Rev Educ Fís. 2007;139:58-70.

23. Piek J. The relationship between bullying and self-worth in children with movement coordination problems. Br J Educ Psychol. 2010;75:453-63.

24. Piek J, Bayanam G, Barrett N. The relationship between fine and gross motor ability, self-perceptions and self-worth in children and adolescents. Hum Mov Sci. 2006;25:65-75.

25. Bejerot S, Humble M. Relevance of motor skill problems in victims of bullying. Pediatrics. 2007:120:1226-7.

26. Campbell W, Missiuna C, Vaillancourt T. Peer victimization and depression in children with and without motor coordination difficulties. Psychol Sch. 2012;49:328-41.

27. Harter S. Developmental processes in the construction of self. In: Yawkey T, Johnson J, editors. Integrative process and socialization: early to middle childhood. Hillsdale: Lawrence Erlbaum; 1988. p.45-78.

28. Schoemaker M, Kalverboer A. Social and affective problems in children who are clumsy: how early do they begin? Adapt Phys Activ Q. 1996;11:130-40.

29. Wittchen HU, Stein MB, Kessler C. Social fears and social phobia in a community sample of adolescents and young adults: prevalence, risk factors and co-morbidity. Psychol Med. 1999:29:309-23.

30. Dewey D. Developmental coordination disorder: associated problems in attention, learning, and psychosocial adjustment. Hum Mov Sci. 2002;21:905-18.

31. Ulrich D. Test of gross motor development: examiner's manual. 2nd ed. Austin: Pro Ed; 2000.

32. Krech D, Crutchfiel RS, Ballachey EL. O indivíduo na sociedade: um manual de psicologia social. São Paulo: Pioneira; 1975.

33. Morais MLS, Otta E. Scala CT. Status sociométrico e avaliaçáo de características comportamentais: um estudo de competência social em pré-escolares. Psicol Reflex Crít. 2001;14:119-31.

34. Coie, JD, Dodge KA, Coppotelli, H. Dimensions and types of social status: a cross-age perspective. Dev Psychol. 1982:18:557-70.

35. Clark J. Motor development. In: Ramachan-Dran V, editor. Encyclopedia of human behavior. San Diego: Academic Press; 1994. p.245-55. 
36. Gallahue D, Donnelly F. Educação física desenvolvimentista para todas as crianças. São Paulo: Phorte; 2008.

37. Palma M, Camargo V, Pontes M. Efeitos da atividade física sistemática sobre o desempenho motor de crianças préescolares. Rev Educ Fís/UEM. 2012;23:421-9.

38. Gaya A. Os jovens atletas brasileiros: relatório do estudo de campo dos jogos da juventude 1996. Brasília: INDESP; 1997.

39. Glaner M. Referenciais para o crescimento físico de adolescentes Gaúchos e Catarinenses. Rev Bras Cineantropom Desempenho Hum. 2003;5:17-26.

40. Esteves AC, Reis DC, Caldeira RM, Leite RM, Moro ARP, Borges Júnior NG. Força de preensão, lateralidade, sexo e características antropométricas da mão de crianças em idade escolar. Rev Bras Cineantropom Desempenho Hum. 2005;7:69-75.

41. Krebs R, Macedo FDO. Desempenho da aptidão física de crianças e adolescentes. Lect Educacion Fisica Deportes. 2005;85. Disponível em: http://www.efdeportes.com/efd85/aptidao.htm.

42. Cieslak F, Levandoski G, Meira Góes S. Relação do nível de qualidade de vida e atividade física em acadêmicos de educaçáo física. Fit Perform J. 2007;6:357-61.

43. Blatchford P, Baines E, Pellegrini A. The social context of school playground games: sex and ethnic difference, and changes over time after entry to junior school. Br J Dev Psychol. 2003;21:481-505.

44. Abiko RH, Caruzzo NM, Bim RH, Nazario PF, Vieira JLL. Avaliação do desempenho motor de crianças de 6 a 9 anos de idade. Cinergis. 2013;13:21-6.

45. Eckert H. Desenvolvimento motor. São Paulo: Manole; 1993.

46. Gabbard C. Lifelong motor development. 3rd ed. Boston: Allyn and Bacon; 2000.

47. Berns R. O desenvolvimento da criança. São Paulo: Loyola; 2002.

48. Rapin I. Disorders of higher cerebral function in preschool children: second of two parts. Am J Dis Child. 1988;142: 1178-82.

49. Selikowitz M. Dislexia e outras dificuldades de aprendizagem. Rio de Janeiro: Revinter; 2001.

50. Nopola-Hemmi J, Myllyluoma B, Voutilainen A, Leinonen S, Kere J, Ahonen T. Familial dyslexia: neurocognitive and genetic correlation in a large Finnish family. Dev Med Child Neurol. 2002;44:580-6.

51. Carvalho MD. Sucesso e fracasso escolar: uma questão de gênero. Educ Pesqui. 2003;29:185-93.

52. Capellini S, Tonelotto J, Ciasca S. Medidas de desempenho escolar: avaliação formais e opinião dos professores. Estud Psicol. 2004;21:79-90.

53. Menezes Filho N. Os determinantes do desempenho escolar do Brasil. São Paulo: IBMEC; 2007.

54. Shaywitz BA, Fletcher JM, Shaywitz SE. Attention-deficit/hyperactivity disorder. Adv Pediatr. 1997;44:331-67.

55. Tirosh E, Berger J, Cohen-Ophir M, Davidovitch M, Cohen A. Learning disabilities with and without attention-deficit hyperactivity disorder: parents' and teachers' perspectives. J Child Neurol. 1998;13:270-6.

56. Brannon L. Gender: psychological perspectives. Boston: Allyn and Bacon; 1999.

57. Ostberg V. Children in classrooms: peer status, status distribution and mental well-being. Soc Sci Med. 2003;56:17-29.

58. Attili, G. Successful and disconfirmed children in the peer group: indices of social competence within an evolutionary perspective. Hum Dev. 1990; 33:238-49.

59. Haywood K, Getchell N. Desenvolvimento motor ao longo da vida. Porto Alegre: Artmed; 2004.

60. Nocentini A, Menesini E, Salmivalli C. Level and change of bullying behavior during high school: a multilevel growth curve analysis. J Adolesc. 2013;36:495-505.

61. Levandoski G, Cardoso F. Imagem corporal e status social de estudantes brasileiros envolvidos em bullying. Rev Latinoam Psicol. 2013;45:135-45.

62. Chase MA, Dummer GM. The role of sports as a social status determinant for children. Res Q Exerc Sport. 1992;63:418-24.

63. Weineck J. Biologia do esporte. 7a ed. São Paulo: Manole; 2005.

64. O’Beirne C, Larkin D, Cable T. Coordination problems and anaerobic performance in children. Adapt Phys Activ Q. 1994;11:141-9.

65. Scarpa S, Carraro A, Gobbi E, Nart A. Peer-victimization during physical education and enjoyment of physical activity. Percept Mot Skills. 2012;115:319-24.

ENDEREÇO

Pâmella Medeiros

Centro de Ciências da Saúde e do Esporte

Universidade do Estado de Santa Catarina

R. Pascoal Simone, 358

88080-350 - Florianópolis - SC - BRASIL e-mail: pamellamedeiros@hotmail.com

Recebido para publicação: 03/03/2014

1a. Revisão: 28/03/2015

2a. Revisão: 10/07/2015

Aceito: 17/07/2015 